\title{
Implementación de Invention System Kits como metodología de enseñanza y aprendizaje en ingeniería industrial. Caso de estudio: Escuela Colombiana de Ingeniería Julio Garavito
}

\author{
Ivonne Angélica Castiblanco-Jiménez ${ }^{a}$, Joan Paola Cruz-González ${ }^{a}$, Carlos Rodrigo Ruiz-Cruz ${ }^{a}$, Liliana del \\ Carmen Pedraza-Vega ${ }^{b}$ \& Diana Catalina Londoño-Restrepo ${ }^{a}$
${ }^{a}$ Decanatura de Ingeniería Industrial, Escuela Colombiana de Ingeniería Julio Garavito, Bogotá, Colombia. ivonne.castiblanco@escuelaing.edu.co, joan.cruz@escuelaing.edu.co, carlosr.ruiz@escuelaing.edu.co,diana.londono@escuelaing.edu.co
${ }^{b}$ Vicerrectoría Académica, Escuela Colombiana de Ingeniería Julio Garavito, Bogotá, Colombia. liliana.pedraza@escuelaing.edu.co

\begin{abstract}
Resumen - Este documento muestra los resultados de un proyecto de investigación cuyo objetivo es explorar nuevas metodologías de enseñanza a través del uso de Invention System Kits, que permitan identificar métodos de aprendizaje en los estudiantes de Ingeniería Industrial de la Escuela Colombiana de Ingeniería Julio Garavito. En particular, se pretende utilizar herramientas de aprendizaje activo como Lego Mindstorms EV3 para potenciar competencias tecnológicas en los estudiantes.
\end{abstract}

Palabras Clave-Ingeniería, metodologías de enseñanza y aprendizaje, invention system kits, lego mindstorms.

Recibido: 18 de enero de 2017. Revisado: 23 de mayo de 2017. Aceptado: 30 de mayo de 2017

Invention System Kits Implementation as teaching and learning methodology in industrial engineering. Case study: Escuela Colombiana de Ingeniería Julio Garavito

Abstract- This paper shows the results of a research project whose objective is to explore new teaching methodologies through the use of Invention System Kits, that allow to identify learning methods for Industrial Engineering students from Escuela Colombiana de Ingeniería Julio Garavito. Particularly, the intention is to use active learning tools such as Lego Mindstorms EV3 to enhance technological competencies in the students.

Keywords - Engineering, Teaching and learning methodologies, invention system kits, lego mindstorms.

\section{Introducción}

El uso de materiales didácticos como Invention Systems Kits ha demostrado ser altamente efectivo como complemento a los procesos de enseñanza y aprendizaje en diversas áreas del conocimiento. Por esta razón se decide implementar como proyecto estas herramientas en la Escuela Colombiana de Ingeniería Julio Garavito en el programa de Ingeniería Industrial, con el objetivo de que los estudiantes adquieran y mejoren conocimientos, capacidades y aptitudes en el campo de la ingeniería.

Haciendo uso de los Invention System Kits, específicamente Lego Mindstorms EV3, es posible generar sistemas para imitar los procesos de ingeniería utilizados en la industria, a través del desarrollo del proyecto se espera que los estudiantes mejoren sus habilidades tecnológicas, además de hacer uso de sus competencias como resolución de problemas, trabajo en equipo y comunicación que no se refuerzan fácilmente con otros métodos.

La metodología plantea replicar, modificar y crear diferentes productos industriales como lo son un seleccionador, un brazo robótico, una fábrica de ensamble de trompos, un sistema de suministro y una ensambladora de aviones; con el fin de generar maneras de pensar que aporten al conocimiento científico, además de identificar, analizar y definir elementos significativos de un problema de manera que se resuelva efectivamente y con un buen criterio.

En primera instancia se muestran los antecedentes encontrados en el campo de investigación de este proyecto y una revisión bibliográfica sobre métodos, modelos o estilos de tanto de enseñanza como de aprendizaje. La siguiente sección muestra la metodología utilizada en el proyecto para posteriormente mostrar los resultados encontrados y las conclusiones y estudios futuros resultantes de su ejecución.

\section{Antecedentes}

En los últimos años, el uso de nuevas herramientas y metodologías de enseñanza se ha hecho extensivo incluyendo el uso de Invention System Kits, ya sea en educación elemental, secundaria o superior. Varios estudios y propuestas se han realizado en torno a este tema. Somyürek [1], muestra cómo mediante el uso de sistemas Lego Mindstorms, los estudiantes logran de una manera dinámica y divertida un aprendizaje activo, auténtico y colaborativo, además de generar múltiples perspectivas para el análisis y solución de problemas; todo como resultado de un entrenamiento en el manejo de estos kits y a la solución de problemas con ayuda de estos.

Como citar este artículo: Castiblanco-Jiménez, I,A., Cruz-González, J.P., Ruiz-Cruz, C.R., Pedraza-Vega, L. del C. and Londoño-Restrepo, D.C., Implementación de Invention System Kits como metodología de enseñanza y wprendizaje en ingeniería industrial. Caso de estudio: Escuela Colombiana de Ingeniería Julio Garavito. Educación en Ingeniería, 12(24), pp. 3137, Julio, 2017. 
Según Scaradozzi et al. [2], el Departamento de Ingeniería de la Información de la Universidad Politécnica de Marche en Italia, con la ayuda del Instituto Comprensivo Largo Cocconi, desarrolló un nuevo programa de educación dirigido a las escuelas primarias de Italia, el cual consiste en el uso de los sistemas Lego WeDo y Lego NXT como complemento de los conceptos científicos y matemáticos normalmente impartidos; esto con el fin de mejorar las habilidades de aprendizaje, resolución de problemas, el trabajo en equipo y motivar el interés de los estudiantes por la tecnología e ingeniería.

Estudios y propuestas similares se han desarrollado dirigidos a estudiantes de secundaria. Tocháček et al. [3] miembros del Departamento de Información, Tecnología y Educación de la Facultad de Educación de la Universidad Charles de Praga en Republica Checa, con el objetivo de que la educación secundaria sea más que la trasferencia de conocimientos y know-how de profesores a estudiantes, desarrollaron un programa para que estos últimos generen nuevos conocimientos y habilidades de su propio trabajo y experiencias mediante proyectos de robótica desarrollados con los sistemas Lego Mindstorms NXT y Lego WeDo.

Por otra parte, en India y Malasia, Balaji et al. [4], bajo la premisa de lo importante que es la ingeniería para el crecimiento y desarrollo de un país, propusieron el uso de los diferentes Invention System Kits de Lego para motivar a los jóvenes a realizar su pregrado en ingeniería, dándoles además habilidades, conocimientos y confianza, necesarios para un excelente desempeño laboral en la industria. El uso de Invention System Kits en la enseñanza de ingeniería es amplio, pues se emplean en diferentes ramas y con diferentes propósitos, tanto pedagógico, como para simular sistemas reales y proponer soluciones a problemas presentes en dichos sistemas.

Müller et al. [5], miembros de la Universidad Técnica de Berlín, Alemania, exploraron la ludificación de los métodos de enseñanza para la dirección de una fábrica mediante el sistema Lego Mindstorms. Para el estudio, los estudiantes trabajaban en grupos con una metodología enfocada a la resolución de problemas, en donde el éxito dependía directamente de la estrategia diseñada mediante el modelo Lego y su funcionalidad, llegando a concluir que los Invention System Kits dan oportunidades para que los estudiantes amplíen sus deseos por explorar. Asimismo, al ser herramientas lúdicas y dinámicas, facilitan el aprendizaje, especialmente cuando los estudiantes tienen un aprendizaje independiente y autodidacta; además de desarrollar competencias sociales, personales y técnicas, los estudiantes mejoran sus habilidades en la planeación y control de sistemas de producción.

En el Departamento de Ciencia Computacional e Inteligencia Artificial de la Universidad de Granada en España, Cuéllar et al. [6], pusieron en práctica técnicas de inteligencia artificial para la resolución de problemas usando los sistemas Lego Mindstorms con estudiantes de tercer año de pregrado en ingeniería computacional. Esta experiencia permitió a los estudiantes mejorar su creatividad, capacidad de abstracción, asimilación de teorías y aplicación de éstas en problemas reales; asimismo, el interés de los estudiantes por la inteligencia artificial incrementó.

Mediante el uso de Invention System Kits, concretamente Lego Mindstorms, Nee et al. [7], procedentes de diversas universidades de Malasia desarrollaron un robot a escala para la inspección de tuberías, capaz de detectar grietas, las cuales están representadas por cintas de color; esto con el fin de usar esta tecnología para la enseñanza y el desarrollo de experimentos a nivel de pregrado. Mediante lo anterior se evidencia cómo el uso de este tipo de herramientas ha sido altamente efectivo, siendo una motivación para continuar generando investigación en este campo.

\section{Marco teórico}

Mucho se ha hablado en literatura sobre métodos, modelos y estilos de aprendizaje, abordados desde puntos de vista tanto pedagógicos como psicológicos. Según Altin et al. [8], los métodos de aprendizaje desarrollados por cada individuo en un contexto educativo son los siguientes: aprendizaje por descubrimiento, aprendizaje colaborativo, aprendizaje basado en proyectos, aprendizaje obligatorio y aprendizaje basado en la competencia. Estos tipos de aprendizaje son mencionados, explicados y validados por otros autores, por ejemplo, Schunk [9] presenta el aprendizaje por descubrimiento y el aprendizaje por colaboración, el primero busca generar conocimiento a través de las nuevas experiencias que se están desarrollando, el segundo afirma que el aprendizaje ocurre cuando los estudiantes trabajan juntos, desarrollando la habilidad de trabajar en equipo.

De igual forma, Pimienta [10] propone algunos de los tipos de aprendizaje planteados por Altin et al. [8] como el aprendizaje por proyectos, que busca que el estudiante se sumerja de manera práctica en una situación o problemática real que requiera de una solución o comprobación; el aprendizaje basado en problemas, que busca que se investigue, proponga y argumente una solución a uno o varios problemas, creando escenarios de posible solución y analizando las posibles consecuencias; y el aprendizaje cooperativo, que propone un aprendizaje mediante equipos de trabajo estructurados y orientados a resolver una tarea específica a través de la colaboración. También presenta el mismo concepto de aprendizaje por colaboración propuesto por Schunk [9].

Kolb [11], también en [12,13], propone un modelo de aprendizaje basado en experiencias, en donde se identifican dos dimensiones principales, la percepción y el procesamiento. En sus estudios encontró que existen dos tipos de percepciones, desde una experiencia directa mediante la acción o desde una experiencia abstracta mediante la teoría. Kolb exploró dos extremos opuestos al hablar de procesamiento, mediante la práctica y experimentación, o mediante la reflexión. De esta forma, propone que el aprendizaje óptimo es el que se desarrolla de manera cíclica en estas cuatro fases: experiencia concreta (actuar), observación reflexiva (reflexionar), conceptualización abstracta (teorizar) y experimentación activa (experimentar). Partiendo de esto, Salas [12] comenta que el aprendizaje es la creación del conocimiento a través de la transformación de una experiencia; es decir que requiere de algún tipo de percepción y de procesamiento.

Al igual que existen diversos estilos de aprendizaje, existen estilos de enseñanza relacionados. Felder et al. [14], también mencionados en [12], proponen estilos de enseñanza y aprendizaje enfocados específicamente al área de la ingeniería, en donde se clasifican y relacionan estos estilos a partir de cinco dimensiones (Ver Tabla 1), se concluye que cada estilo de aprendizaje tiene un estilo de enseñanza asociado. 
Tabla 1

Dimensiones de los estilos de aprendizaje y enseñanza

\begin{tabular}{|c|c|c|c|}
\hline \multicolumn{2}{|c|}{$\begin{array}{c}\text { Estilo de aprendizaje del } \\
\text { estudiante }\end{array}$} & \multicolumn{2}{|c|}{$\begin{array}{c}\text { Estilo de enseñanza que le } \\
\text { corresponde }\end{array}$} \\
\hline Percepción & $\begin{array}{c}\text { Sensitiva } \\
\text { Intuitiva }\end{array}$ & Contenido & $\begin{array}{l}\text { Concreto } \\
\text { Abstracto }\end{array}$ \\
\hline Input & $\begin{array}{l}\text { Visual } \\
\text { Verbal }\end{array}$ & Presentación & $\begin{array}{l}\text { Visual } \\
\text { Verbal }\end{array}$ \\
\hline Organización & $\begin{array}{l}\text { Inductiva } \\
\text { Deductiva }\end{array}$ & Organización & $\begin{array}{l}\text { Inductiva } \\
\text { Deductiva }\end{array}$ \\
\hline Procesamiento & $\begin{array}{c}\text { Activo } \\
\text { Reflexivo }\end{array}$ & $\begin{array}{l}\text { Participación } \\
\text { del estudiante }\end{array}$ & $\begin{array}{l}\text { Activa } \\
\text { Pasiva }\end{array}$ \\
\hline Comprensión & $\begin{array}{l}\text { Secuencial } \\
\text { Global }\end{array}$ & Perspectiva & $\begin{array}{c}\text { Secuencial } \\
\text { Global }\end{array}$ \\
\hline
\end{tabular}

Fuente: Adaptado de [14].

Percepción/Contenido. El estilo sensitivo/concreto es práctico, orientado hacia los hechos y procedimientos, mientras el intuitivo/abstracto es conceptual, orientado hacia las teorías y significados. El proyecto se centra en un enfoque concreto, mediante los hechos evidenciados a través de la experimentación con los sistemas creados con los Invention System Kits.

Input/Presentación. En el estilo visual la información se brinda mediante representaciones visuales, en el verbal la información se da de forma escrita y hablada. En el presente estudio la información se presenta a través de los dos estilos, ya que son altamente complementarios y de fácil acoplamiento; de forma visual mediante la ejemplificación a través de la herramienta y esquemas, o de forma escrita o hablada con guías e instrucciones claras.

Organización. El estilo inductivo se refiere a la inferencia de principios o generalidades a partir de hechos y observaciones; el deductivo a la deducción de consecuencias y aplicaciones a partir de fundamentos o generalidades. El uso de Invention System Kits permite evidenciar tanto el estilo inductivo como el deductivo, pues se infieren principios a partir de la observación y experimentación, además de la aplicación y análisis de teorías para la creación y adecuación de sistemas.

Procesamiento/Participación del estudiante. En el estilo activo los estudiantes tienden a retener y comprender nueva información si hacen algo con ella (discutiéndola, aplicándola o explicándola a otro), lo que genera alta participación; en el reflexivo/pasivo los estudiantes tienden a retener y comprender nueva información pensando y reflexionando sobre ella, lo que genera poca participación. En el proyecto los estudiantes demuestran una participación activa, pues son ellos quienes entran en contacto con la herramienta para aplicar, adquirir y generar conocimiento en la creación de nuevos sistemas.

Comprensión/Perspectiva. Para el estilo secuencial el conocimiento se aprende o se expone en pequeños pasos incrementales siempre lógicamente relacionados y secuenciados; para el global, el conocimiento se adquiere o se expone a grandes saltos y casi al azar hasta lograr visualizar la totalidad de los conceptos o problemas. Para este estudio se busca principalmente un estilo secuencial, mediante la aplicación de la metodología propuesta.

Por otra parte, Ertmer et al. [15] y Urbina [16] mencionan modelos de enseñanza y aprendizaje basados en el conductismo y el constructivismo. En el conductismo el docente juega un papel crucial, compartiendo y dando todo su conocimiento al estudiante para que éste demuestre respuestas apropiadas a ciertos estímulos, como casos o problemas; el estudiante es receptor de conocimiento con el fin de lograr un objetivo de aprendizaje en donde muestre el producto de su aprendizaje, el cual es evaluable, medible y cuantificable. Por otra parte, en el constructivismo el estudiante enriquece sus conocimientos y crea significados mediante la experimentación activa acompañados por el docente, quien tiene el rol de guía; el objetivo de aprendizaje es de carácter abierto y flexible y se evalúa de forma continua y permanente. A pesar de que ambos modelos parecen opuestos, poseen fronteras borrosas y tienden a estar relacionados y ser complementarios, pues las desventajas de uno son las ventajas del otro.

\section{Metodología}

Para la ejecución de este proyecto se seleccionó un grupo de estudiantes de Ingeniería Industrial de la Escuela Colombiana de Ingeniería Julio Garavito, quienes entraron en contacto por primera vez con los Invention System Kits Lego Mindstorms EV3 usados para este proyecto. Por esta razón, la primera fase del desarrollo de este caso de estudio fue enfocada a la familiarización de los estudiantes con la herramienta. Posteriormente, se buscó aplicar este conocimiento sobre el manejo de la herramienta en el desarrollo de proyectos relacionados a sistemas de producción y manufactura, los cuales son de interés e importancia en los procesos de formación de un ingeniero industrial. Al seleccionar los proyectos a realizar se evaluaron las áreas potenciales de aplicación de estas metodologías con base en el plan de estudios de pregrado de Ingeniería Industrial, buscando lograr el mayor impacto en su aprendizaje.

Para explorar nuevas metodologías de enseñanza a través del uso de Invention System Kits e identificar el nivel de aprendizaje alcanzado por cada estudiante, se propusieron tres niveles en relación con la complejidad del proyecto (Ver Tabla 2). El primero corresponde a la construcción y programación mediante la réplica de sistemas anteriormente diseñados con esta herramienta; el segundo, implica la modificación y combinación de sistemas replicados para lograr sistemas más complejos y robustos; el tercero, corresponde a la construcción y automatización de sistemas completamente nuevos.

De igual forma, para el desarrollo de este proyecto, con el objetivo de explorar el uso de nuevas metodologías de enseñanza, y con base en la teoría propuesta por [8] sobre los tipos de aprendizaje, se realizó uso de dos fuentes de información. La primera proveniente del autoconocimiento del estudiante. Para esto se tuvo en cuenta las opiniones de los propios estudiantes quienes realizaron una bitácora y un cuestionario. En la bitácora

Tabla 2

Niveles de aprendizaje propuestos para el caso de estudio

\begin{tabular}{lc}
\hline \multicolumn{1}{c}{ Nivel } & Descripción \\
\hline 1. Réplica & $\begin{array}{c}\text { Construcción y programación de sistemas } \\
\text { anteriormente propuestos. } \\
\text { 2. Adecuación } \\
\text { 3. Creación }\end{array}$ \\
$\begin{array}{c}\text { Modificación y combinación de sistemas y } \\
\text { programas replicados. }\end{array}$ \\
Diseño, creación y automatización se sistemas \\
completamente nuevos.
\end{tabular}

Fuente: Los autores. 
consignaban su opinión y descubrimientos del proceso que estaban llevando a cabo y en el cuestionario, siguiendo lo propuesto por [1], se indagaba de manera más precisa la percepción que tuvieron los estudiantes durante el desarrollo de las diferentes actividades que se les fueron asignadas y los retos que tuvieron en su ejecución. La segunda, proveniente de la observación del profesor, para lo cual los docentes líderes del proyecto con su experiencia en el área de la educación y la ingeniería, evaluaban el aprendizaje de los estudiantes a la luz de la mejora de sus competencias tecnológicas en el ambiente lúdico que se generaba.

Con el fin de tener registro de los progresos y sistemas creados para la implementación de estas nuevas metodologías en el currículo de Ingeniería Industrial, se construyeron protocolos orientados a mejorar el desarrollo de las capacidades tecnológicas de los estudiantes utilizando Invention System Kits, los cuales fueron diseñados y probados por docentes y estudiantes involucrados en este proyecto.

\section{Resultados}

Durante la ejecución del proyecto y la aplicación de la metodología se construyeron diversos sistemas, cada uno con características diferentes, que permitieron explorar los niveles mencionados y generar hallazgos importantes que se explican a continuación.

\subsection{Nivel 1. Réplica}

Se construyó un sorter que es un sistema de clasificación de productos por colores. También se construyó un brazo robótico con características y programación similares, esto contemplado dentro del primer nivel de dificultad. También como réplica, se elaboró una fábrica de spinners como se muestra en la Fig. 1, para lo cual fue necesario profundizar nuevos conocimientos en los cuales un ingeniero industrial no está especializado, como lo son las compuertas lógicas para el uso de transferencia y validación de datos reportados por los sensores, siendo este un conocimiento necesario para un ingeniero industrial que desea incursionar en el diseño y automatización de plantas industriales. Aunque esta temática no se evidencia en el transcurso de la carrera, se torna fácil de aprender y retener mediante ayudas didácticas, dado que al mismo tiempo que se investigaban sobre estas, se podía ver en acción su uso práctico en una fábrica.

\subsection{Nivel 2. Adecuación}

La adecuación de los productos anteriormente mencionados además de ayudar a los estudiantes a alcanzar el segundo nivel de dificultad, ayudó a familiarizarse con el sistema, impulsó el uso de este, la creatividad, el trabajo en equipo, la investigación y la motivación por aprender y seguir desarrollando nuevos proyectos.

\subsection{Nivel 3. Creación}

Dentro de los nuevos diseños de los productos enfocados a la formación del ingeniero industrial, que permitieron una

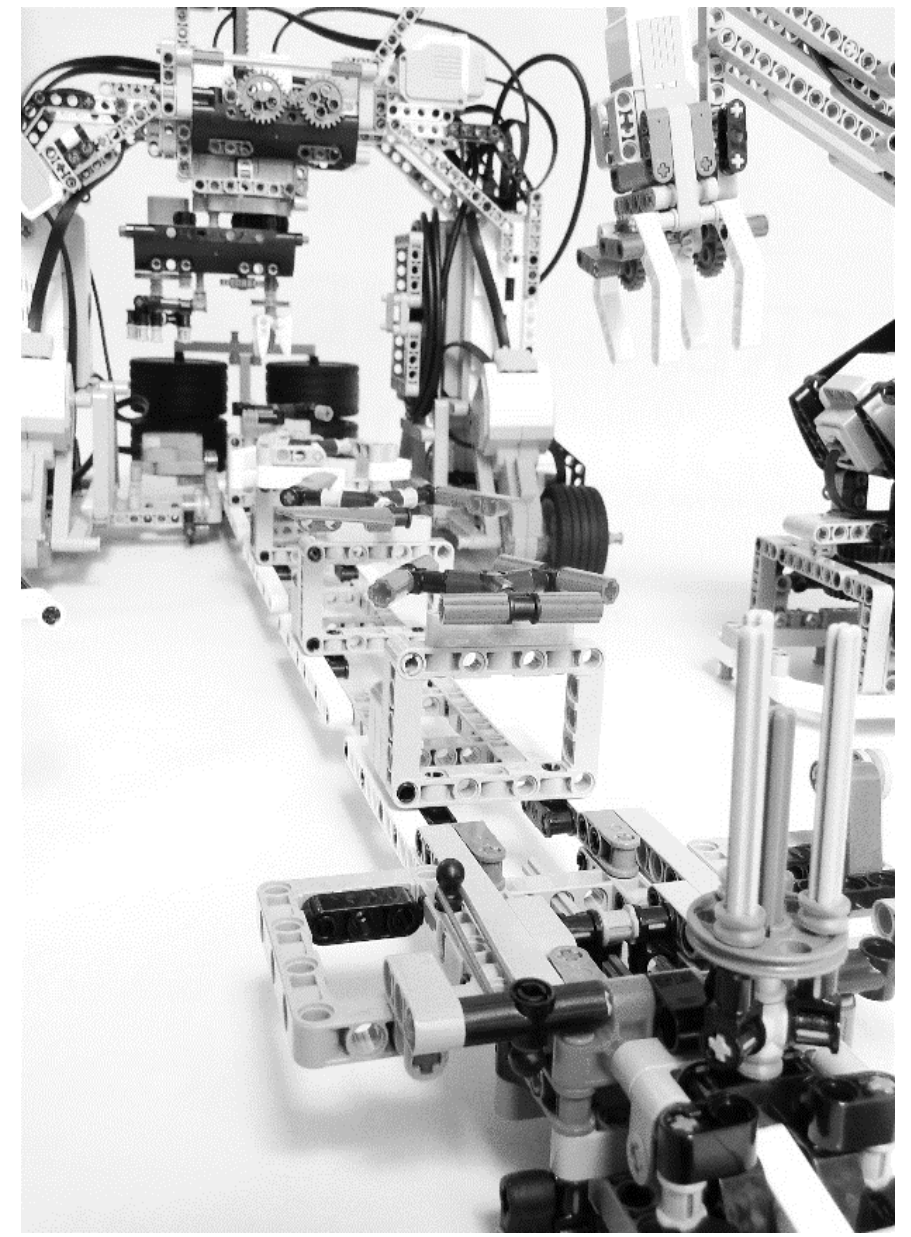

Figura 1. Fábrica de spinners (trompos) y brazo robótico construido. Fuente: Los autores.

transición del segundo al tercer nivel, se construyó un sistema de suministro a partir de una demanda histórica dada, como se observa en la Fig. 2. El aprovisionamiento se realiza por medio de una banda transportadora y tres (3) distribuidores semi-automáticos y las órdenes son actualizadas mediante el uso de sensores. Este sistema permitió ligar el Lego Mindstorms EV3 al software de programación Matlab, generando diversas formas de adecuar y mejorar el funcionamiento del sistema, además de brindar nuevas y variadas oportunidades de aprendizaje para los estudiantes.

Igualmente se construyó una línea de producción de aviones hechos con bloques clásicos de Lego como se presenta en la Fig. 3, siendo esta la primera vez que se incorporaron elementos ajenos al Lego Mindstorms EV3, permitiendo crear un sistema para el ensamble de bloques de Lego tradicionales. Mediante el uso de sensores y compuertas lógicas, el sistema integra una banda transportadora, un brazo robótico y prensas, que funcionan de forma automática una vez se pulsa un interruptor, y sistemas de suministro, que funcionan de forma semi-automática. El desarrollo exitoso de estos retos por parte de los estudiantes, permitió comprobar el perfeccionamiento de las habilidades tecnológicas adquiridas mediante los proyectos realizados anteriormente, además de la mejora de competencias como resolución de problemas, trabajo en equipo, comunicación y generación de nuevos diseños, resultados que se esperaban al inicio de este proyecto. 


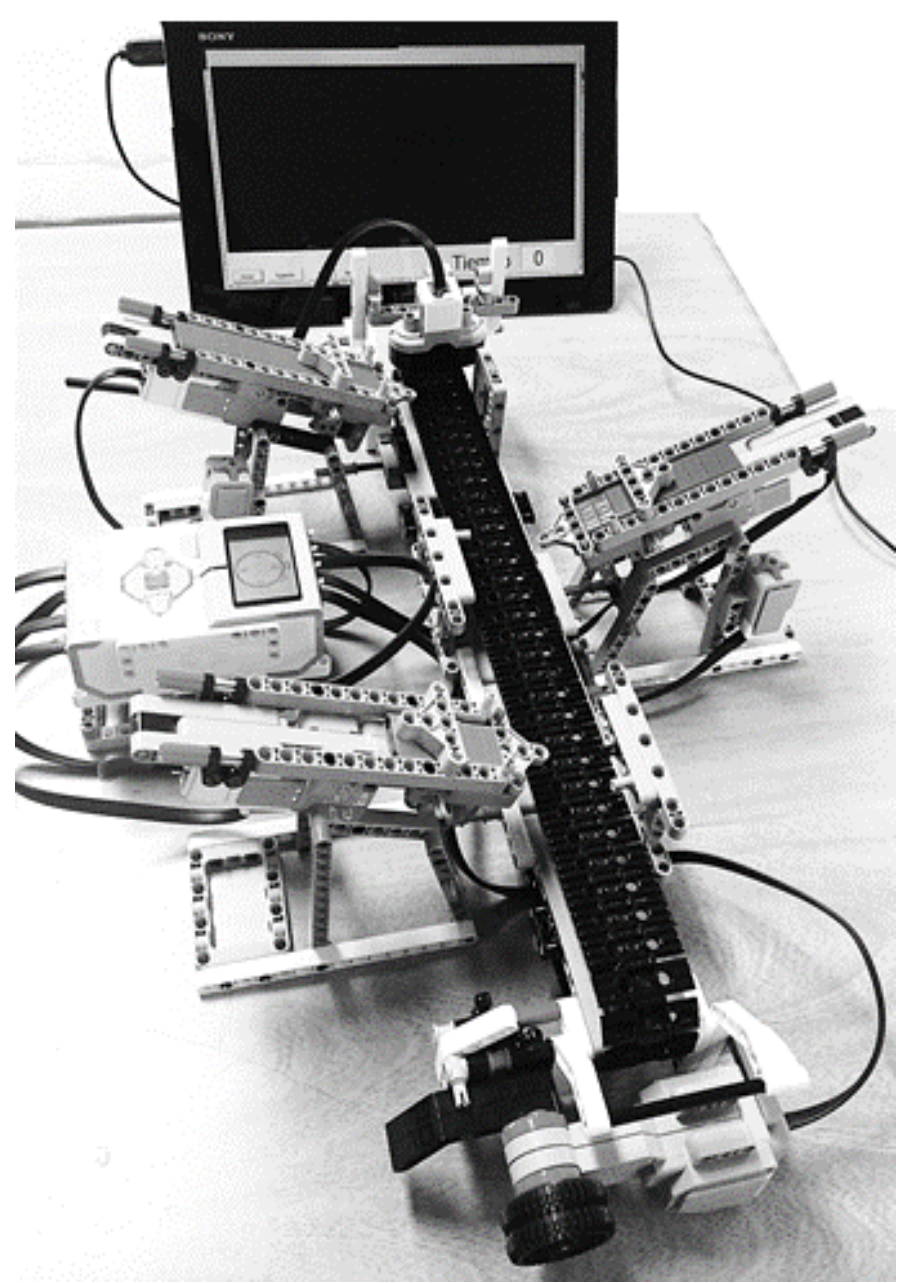

Figura 2. Sistema de suministro a través de banda transportadora y distribuidores semi-automáticos.

Fuente: Los autores.

\subsection{Análisis de Resultados}

Durante el desarrollo de la metodología y como resultado de las observaciones, los métodos de aprendizaje planteados por [8] que se han evidenciado en el proyecto son:

\subsubsection{Aprendizaje por descubrimiento}

Los estudiantes interactuaron con los Invention System Kits y pudieron aprender acerca de programación y construcción de estructuras mediante una exploración propia con el material disponible, la experimentación con la herramienta y el uso del método de prueba y error.

\subsubsection{Aprendizaje colaborativo}

Se activó el proceso de comunicación entre los integrantes del grupo al compartir la elaboración de una misma tarea, permitiendo la colaboración al dividir una labor principal en algunas secundarias y al comunicar ideas y conocimientos para la solución de los diversos problemas que se presentaron en el desarrollo de los proyectos.

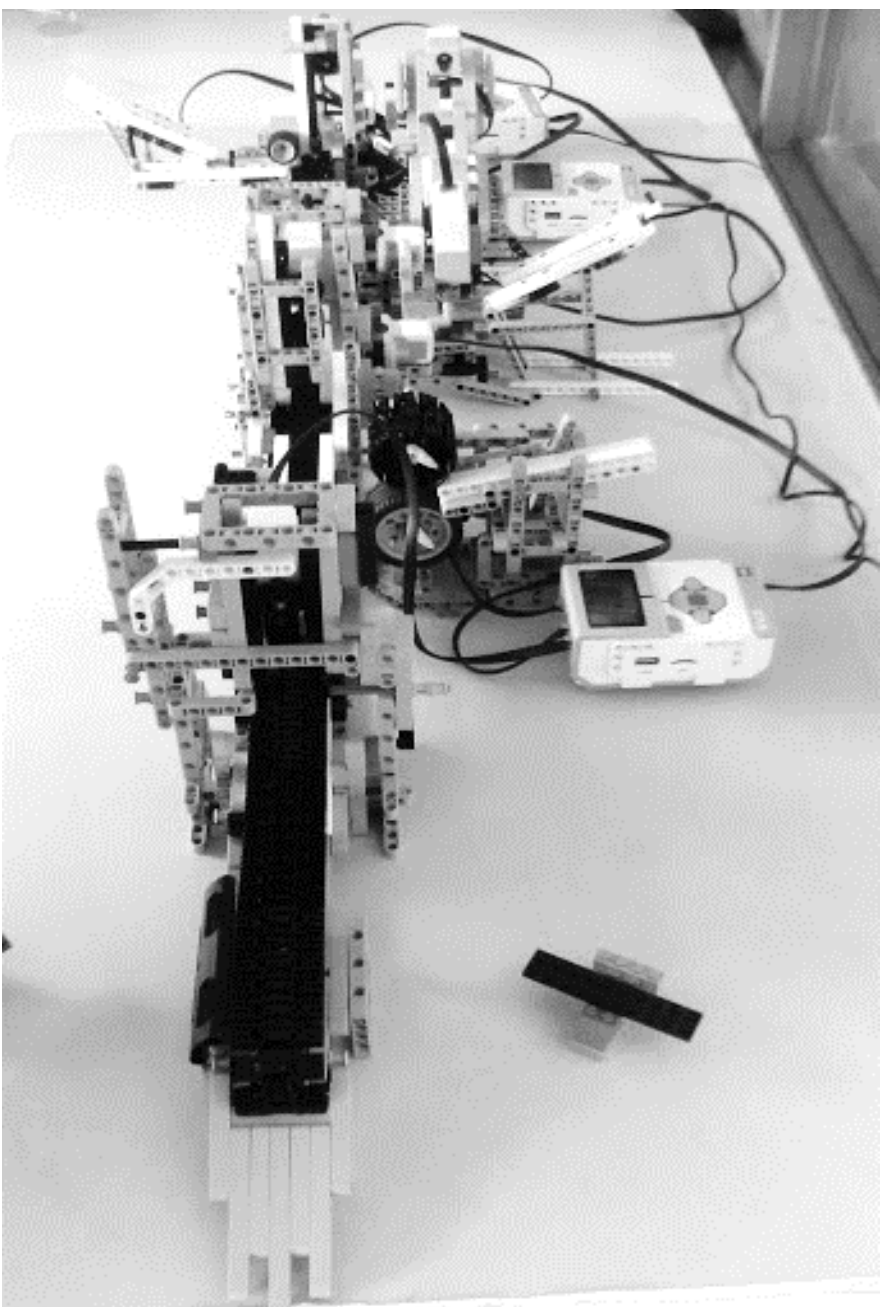

Figura 3. Línea de ensamble de aviones. Fuente: Los autores.

\subsubsection{Aprendizaje basado en proyectos}

Se realizó la construcción de los productos industriales anteriormente mencionados, así como el análisis y desarrollo de la programación requerida, evidenciando de esta manera un ciclo de aprendizaje continuo, pues cada proyecto además de permitir aplicar los conocimientos adquiridos en otros proyectos, permitía la adquisición de nuevos conocimientos y el uso de diferentes métodos de análisis y solución.

Adicionalmente, durante la interacción con Lego Mindstorms EV3 y la realización de los productos industriales, se pudo evidenciar que cada estudiante tiene procesos de aprendizaje y de interacción diferentes. Algunas personas prefieren trabajar de manera individual y otras de manera grupal; igualmente algunos prefieren profundizar sus conocimientos teóricos mediante la investigación para poder enfrentarse a un problema, mientras que otros prefieren abordar el problema directamente y aplicar métodos como prueba y error, evidenciando los estilos de aprendizaje mencionados por [14]. Por lo cual, así como menciona [14], independientemente de los métodos propios de cada estudiante, es posible hacer uso de diversos métodos de enseñanza que permitan mejorar su aprendizaje individual y grupal. 
$\mathrm{Al}$ evaluar las posibilidades de aplicación de los Invention System Kits dentro del plan de estudios de Ingeniería Industrial, se evidenció que esta herramienta puede ser usada en diversas áreas de este, además de reforzarlo y complementarlo, pues permite modelar temas como diseño de productos y procesos, balanceo de línea, estudio de tiempos, conceptos de física mecánica, automatización, entre otros. Para poder aplicar la herramienta en dichas áreas se generaron protocolos para introducir a los estudiantes en la creación, automatización y análisis de sistemas. Según los métodos de enseñanza propuestos por [14], mediante el uso de los protocolos se busca que los estudiantes repliquen diversos sistemas en un trabajo guiado por el docente con perspectiva secuencial, brindando una enseñanza de contenido concreto, en donde la organización de información es de forma tanto inductiva como deductiva; la generación de conocimientos se logra mediante la práctica y la participación activa de los estudiantes.

En el desarrollo de los proyectos y la creación y diseño de protocolos se evidencia el ciclo de aprendizaje optimo propuesto por [11] como se muestra en la Fig. 4. Inicialmente los estudiantes tienen cierta conceptualización y conocimiento sobre los temas referentes a su formación como ingenieros industriales, posteriormente proceden a tener contacto y experimentar con los Invention System Kits, generando experiencias concretas a través del uso de la herramienta y creación de sistemas. Estas experiencias permiten la reflexión y la creación de nuevo conocimiento que es analizado y evaluado con el fin de ser plasmado en protocolos, en los cuales combinan el conocimiento anterior y el nuevo conocimiento, generando una guía que sirve como input en el aprendizaje de terceros, dando así continuidad al ciclo y a la oportunidad de generar nuevos proyectos.

A partir de todo lo expuesto anteriormente, se observa que en el proyecto los estudiantes tuvieron una participación constante y activa, siendo ellos los protagonistas y el centro del proceso y los autores de su propio aprendizaje a través de experiencias, mientras que los docentes adquirieron un papel de guía y apoyo en el proceso, facilitando la adquisición de conocimiento por parte del estudiante, según esto y lo propuesto por $[15,16]$ en este proyecto se

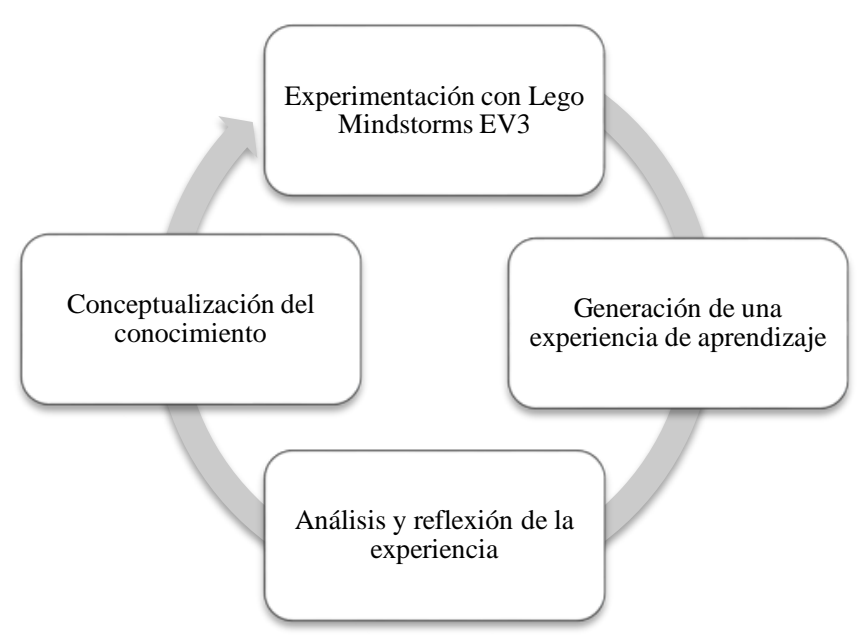

Figura 4. Ciclo de aprendizaje de Kolb [11] evidenciado en el caso de estudio. Fuente: Los autores. presenta un modelo de enseñanza basado principalmente en el constructivismo. Aunque el aprendizaje se genere de forma autónoma y el estudiante construya su propio conocimiento mediante la experiencia, no se puede olvidar que se mantienen elementos de un modelo conductista, pues al final la generación y evaluación de resultados es la forma de medir y comprobar la consecución de un objetivo de aprendizaje, el cual esta previamente establecido como parte de un currículo para la formación de ingenieros industriales.

\section{Conclusiones}

Después de haber analizado el proceso de aprendizaje de los estudiantes a través de la aplicación de la metodología propuesta, se evidenció que efectivamente los Invention System Kits, en particular Lego Mindstorms EV3, permiten el aprendizaje activo de los estudiantes de Ingeniería. El enfoque propuesto se realizó de forma secuencial, lo que ayudó a los estudiantes a generar un aprendizaje progresivo y lógico facilitando así la utilización de una estrategia constructivista como metodología de enseñanza y permitiendo generar ciclos de aprendizaje claros y efectivos.

Gracias a la dinamización de las metodologías de aprendizaje, se facilitó la familiarización con la herramienta, además de impulsar su uso, aumentar la creatividad, la investigación, el compromiso y la motivación, importantes para el desarrollo de estilos de aprendizaje autónomos por parte de cada estudiante; adicionalmente se evidenció la mejora de habilidades tecnológicas y competencias como resolución de problemas, trabajo en equipo y comunicación en los estudiantes.

La generación de protocolos es una herramienta útil para la trasferencia de conocimientos, dando pasos e instrucciones claras que permitan un aprendizaje autónomo y secuencial en el estudiante, por eso puede incorporarse en el plan de estudios de un programa de Ingeniería Industrial, con el fin de complementar y reforzar el aprendizaje teórico y técnico de los estudiantes.

\subsection{Estudios futuros}

En el futuro, además de aplicar y comprobar la eficiencia de este tipo de metodologías en grupos de mayor tamaño y con una mayor variedad de estudiantes, se desea la incorporación de nuevas herramientas diferentes al Lego Mindstorms EV3, que en conjunto se complementen y permitan abarcar una mayor cantidad de temáticas que generen impactos importantes y significativos para la formación de ingenieros industriales.

\section{Agradecimientos}

Especial agradecimiento a los estudiantes que se han vinculado voluntariamente al semillero de Producción, para trabajar en este proyecto, por su arduo trabajo y compromiso:

Carlos Mauricio Urrego. Estudiante Ingeniería Industrial. Escuela Colombiana de Ingeniería Julio Garavito. Bogotá, Colombia.

Juan Sebastián Cadena Muñoz. Estudiante Ingeniería Industrial. Escuela Colombiana de Ingeniería Julio Garavito. Bogotá, Colombia. 
Miguel Ángel Barragán Cantor. Estudiante Ingeniería Industrial. Escuela Colombiana de Ingeniería Julio Garavito. Bogotá, Colombia.

\section{Referencias}

[1] Somyürek, S., An effective educational tool: Construction kits for fun and meaningful learning, International Journal of Technology and Design Education., 25, pp. 25-41, 2015. DOI: 10.1007/s10798-0149272-1

[2] Scaradozzi, D., Sorbi, L., Pedale, A., Valzano, M. and Vergine, C., Teaching robotics at the primary school: An innovative approach, Procedia - Social Behavioral Sciences, 174, pp. 3838-3846, 2015. DOI: 10.1016/j.sbspro.2015.01.1122

[3] Tocháček, D., Lapeš, J. and Fuglík, V., Developing technological knowledge and programming skills of secondary schools students through the educational robotics projects, Procedia - Social and Behavioral Sciences, 217, pp. 377-381, 2016. DOI: 10.1016/j.sbspro.2016.02.107

[4] Balaji, M., Balaji, V., Chandrasekaran, M., Khan, M.K.A.A. and Elamvazuthi, I., Robotic training to bridge school students with engineering, Procedia Computer Science, 76, pp. 27-33, 2015. DOI: 10.1016/j.procs.2015.12.271

[5] Müller, B.C., Reise, C. and Seliger, G., Gamification in factory management education - A case study with LEGO Mindstorms, Procedia CIRP, 26, pp. 121-126, 2015. DOI: 10.1016/j.procir.2014.07.056

[6] Cuéllar M.P. and Pegalajar, M.C., Design and implementation of intelligent systems with LEGO Mindstorms for undergraduate computer engineers, Computer Applications in Engineering Education, 22, pp. 153-166, 2014. DOI: 10.1002/cae.20541

[7] Nee, L.V., Elamvazuthi, I., Ganesan, T., Khan, M.K.A.A. and Parasuraman, S., Development of a laboratory-scale pipeline inspection robot, Procedia Computer Science, 76, pp. 9-14, 2015. DOI: 10.1016/j.procs.2015.12.268

[8] Altin, H. and Pedaste, M., Learning approaches to applying robotics in science education, Journal of Baltic Science Education, 12, no. 3, pp. 365-370, 2013.

[9] Schunk, D.H., Learning theories: An educational perspective. Boston: Pearson, 2012.

[10] Pimienta-Prieto, J.H., Estrategias de enseñanza-aprendizaje: Docencia universitaria basada en competencias. Naucalpan de Juárez: Pearson, 2012.

[11] Kolb, D.A., Experiential learning: Experience as the source of learning and development. Nueva Jersey: Prentice-Hall, 1984.

[12] Salas-Silva, R.E., Estilos de aprendizaje a la luz de la neurociencia. Bogotá D.C: Cooperativa Editorial Magisterio, 2008.

[13] Aragón-de Viau, M., Estilos de aprendizaje. Guatemala: Universidad Rafael Landívar, 2000.

[14] Felder, R.M. and Silverman L.K., Learning and teaching styles in engineering education, Journal of Engineering Education, 78(7), pp. 674681, 1988.

[15] Ertmer, P.A. y Newby, T.J., Conductismo, cognitivismo y constructivismo: Una comparación de los aspectos críticos desde la perspectiva del diseño de instrucción, Performance Improvement Quarterly, 6(4), pp. 50-72, 1993.

[16] Urbina-Ramírez, S., Informática y teorías del aprendizaje, Pixel-Bit. Revista de Medios y Educación, 12, pp. 87-100, 1999.
I.A. Castiblanco-Jiménez, es Ing. Electrónica en 2011 de la Pontificia Universidad Javeriana, Bogotá, Colombia, MSc. en Ingeniería Mecatrónica en 2011 del Politécnico di Torino, Turín, Italia, MSc. en Automatización Industrial en 2014 del Politécnico di Torino, Turín, Italia. Esp. en Lean Manufacturing y World Class Manufacturing. Experiencia en el campo de gestión de instalaciones, desarrollo de producto y producción para el grupo FIAT CHRYSLER en Turín, Italia. Profesor de planta de la Escuela Colombiana de Ingeniería Julio Garavito en el programa de Ingeniería Industrial. Centro de Investigaciones en Manufactura y Servicios - CIMSER, Bogotá, Colombia. ORCID: 0000-0001-5866-078X

J.P. Cruz-González, es Ing. Industrial en 2007 de la Universidad de los Andes, Bogotá, Colombia, MSc. en Ingeniería Industrial en el Área de Gestión y Dirección Organizacional en 2009 de la Universidad de los Andes, Bogotá, Colombia. Experiencia como consultora en diferentes multinacionales y profesor de cátedra en la Universidad de los Andes. Profesor de planta de la Escuela Colombiana de Ingeniería Julio Garavito en los programas de Ingeniería Industrial y MSc. en Ingeniería Industrial. Directora del Centro de Estudios de Sistemas de Gestión. Centro de Investigaciones en Manufactura y Servicios - CIMSER, Bogotá, Colombia.

ORCID: 0000-0002-5343-9055

C.R. Ruiz-Cruz, es Ing. Industrial en el año 2000 de la Escuela Colombiana de Ingeniería Julio Garavito, Bogotá, Colombia, Esp. en Logística de la Universidad del Valle, Cali, Colombia, en 2006 y MSc. en Ingeniería de esta última universidad en 2008. Profesor de planta del programa de Ingeniería Industrial de la Escuela Colombiana de Ingeniería Julio Garavito. Director del Centro de Investigaciones en Manufactura y Servicios - CIMSER, Bogotá, Colombia.

ORCID: 0000-0002-0671-7382

L.C. Pedraza-Vega, es Ing. de Sistemas en 1996 de la Escuela Colombiana de Ingeniería Julio Garavito, Bogotá, Colombia, coordinadora de desarrollo profesoral de la Escuela Colombiana de Ingeniería Julio Garavito. Vicerrectoría Académica. Innovación Docente e Investigación Educativa - INNOVAD-IE, Bogotá, Colombia.

ORCID: 0000-0003-4725-5927

D.C. Londoño-Restrepo, es Ing. de Producción en 2002 de la Universidad EAFIT, Medellín, Colombia, Esp. en Logística Internacional en 2008 de ESUMER, Medellín, Colombia y MSc. en Administración de Empresas con Especialidad en Gestión Integrada de la Calidad, Seguridad y Medio Ambiente en 2012 de la Universidad de Viña del Mar, Chile. Profesor de planta del programa de Ingeniería Industrial de la Escuela Colombiana de Ingeniería Julio Garavito. Centro de Investigaciones en Manufactura y Servicios - CIMSER, Bogotá, Colombia.

ORCID: 0000-0001-5052-0889 